\title{
BMJ Open Physical activity, sedentary time and their associations with clustered metabolic risk among people with type 2 diabetes in Jiangsu province: a cross- sectional study
}

\author{
Yijia Chen, ${ }^{1,2}$ Jie Yang, ${ }^{2}$ Jian Su, ${ }^{2}$ Yu Qin, ${ }^{2}$ Chong Shen, ${ }^{3}$ Ying Li, ${ }^{1}$ Shurong Lu, ${ }^{2}$ \\ Enchun Pan, ${ }^{4}$ Yan Gao, ${ }^{5}$ Dandan Miao, ${ }^{4}$ Ning Zhang, ${ }^{6}$ Jinyi Zhou, ${ }^{1,2}$ Ming Wu ${ }^{1,2}$
}

To cite: Chen Y, Yang J, Su J, et al. Physical activity, sedentary time and their associations with clustered metabolic risk among people with type 2 diabetes in Jiangsu province: a crosssectional study. BMJ Open 2019;9:e027906. doi:10.1136/ bmjopen-2018-027906

- Prepublication history and additional material for this paper are available online. To view please visit the journal (http:// dx.doi.org/10.1136/bmjopen2018-027906).

YC and JY contributed equally.

Received 16 November 2018

Revised 28 June 2019

Accepted 19 July 2019

Check for updates

(C) Author(s) (or their employer(s)) 2019. Re-use permitted under CC BY-NC. No commercial re-use. See rights and permissions. Published by BMJ.

For numbered affiliations see end of article.

Correspondence to Professor Ming Wu; jswuming@vip.sina.com

\section{ABSTRACT}

Objective Investigating the association between total physical activity, physical activity in different domains and sedentary time with clustered metabolic risk in patients with type 2 diabetes from Jiangsu province, China.

Design Interview-based cross-sectional study conducted between December 2013 and January 2014.

Setting 44 selected townships across two cities, Changshu and Huai'an, in Jiangsu province.

Participants 20340 participants selected using stratified cluster-randomised sampling and an interviewer-managed questionnaire.

Methods We constructed clustered metabolic risk by summing sex-specific standardised values of waist circumference, fasting triacylglycerol, fasting plasma glucose, systolic blood pressure and the inverse of blood high-density lipoprotein cholesterol (HDL-cholesterol). Self-reported total physical activity included occupation, commuting and leisure-time physical activity. The unstandardised regression coefficient $[\mathrm{B}]$ and its $95 \% \mathrm{Cl}$ were calculated using multivariate linear regression analyses.

Results This study included 17750 type 2 diabetes patients (aged 21-94 years, $60.3 \%$ female). The total ( $\mathrm{B}=-0.080 ; 95 \% \mathrm{Cl}:-0.114$ to -0.046$)$, occupational $(\mathrm{B}=-0.066 ; 95 \% \mathrm{Cl}:-0.101$ to -0.031$)$ and leisuretime physical activity $(\mathrm{B}=-0.041 ; 95 \% \mathrm{Cl}:-0.075$ to $-0.007)$, and sedentary time $(\mathrm{B}=0.117 ; 95 \% \mathrm{Cl}: 0.083$ to 0.151 ) were associated with clustered metabolic risk. Total physical activity, occupational physical activity and sedentary time were associated with waist circumference, triacylglycerol and HDL-cholesterol, but not with systolic blood pressure. Commuting physical activity and sedentary time were significantly associated with triacylglycerol $(\mathrm{B}=-0.012 ; 95 \% \mathrm{Cl}:-0.019$ to -0.005$)$ and fasting plasma glucose ( $\mathrm{B}=0.008 ; 95 \% \mathrm{Cl}: 0.003$ to 0.01$)$, respectively. Leisure-time physical activity was only significantly associated with systolic blood pressure $(B=-0.239$; 95\% Cl: -0.542 to- 0.045).

Conclusions Total, occupational and leisure-time physical activity were inversely associated with clustered metabolic risk, whereas sedentary time increased metabolic risk. Commuting physical activity was inversely associated with triacylglycerol. These findings suggest that increased

\section{Strengths and limitations of this study}

- This study constructed clustered metabolic risk by summing sex-specific standardised values for waist circumference, fasting triacylglycerol, fasting plasma glucose, systolic blood pressure and the inverse of high-density lipoprotein cholesterol.

- The study has a relatively large sample size of 17750 participants, all of whom are community-managed patients with type 2 diabetes in China.

- The study investigated physical activity in different domains and sedentary time and their associations with metabolic risk factors.

- The limitation of this study was that information regarding physical activity and sedentary time was collected through the use of a self-reporting questionnaire.

physical activity in different domains and decreased sedentary time may have protective effects against metabolic risk in type 2 diabetes patients.

\section{INTRODUCTION}

The clustering of metabolic risk factors for cardiovascular disease and type 2 diabetes mellitus is known as metabolic syndrome. ${ }^{1}$ Based on the National Cholesterol Education Program definition, ${ }^{2}$ metabolic syndrome is a rapidly growing epidemic in China. A meta-analysis estimated that the pooled prevalence of metabolic syndrome in mainland China was $24.5 \%,{ }^{3}$ but was $55.7 \%$ among patients with type 2 diabetes. ${ }^{4}$ Individuals with metabolic syndrome were found to be at an increased risk of developing diabetes, cardiovascular disease and all-cause mortality. ${ }^{5-7}$ This increasing prevalence among Chinese populations and its severe health consequences has led to a call for prevention and 
maintenance strategies, and the improvement of metabolic health in one of the largest populations in the world.

Physical activity has long been considered as a key factor in the prevention and management of metabolic risk factors. ${ }^{8}$ A number of studies have investigated the association between physical activity and glycaemic control, ${ }^{9}$ lipid profile ${ }^{10}$ and clustered metabolic risk. ${ }^{11} 12$ Most emphasised the value of assessing overall physical activity or leisure-time physical activity, ${ }^{9-12}$ but few have focused on other domains of physical activity (eg, occupational, household or commuting) ${ }^{13}$ Information on different domains of physical activity may be crucial for Chinese people since they have been found to have quite different patterns of physical activity compared with their Western counterparts. For example, it has been reported that occupational and household activities contribute substantially more to total physical activity than leisuretime or transportation activity. ${ }^{14}$ Therefore, it is important to examine whether other types of physical activities, such as occupational and commuting physical activity, are associated with metabolic risk among patients with diabetes.

Emerging evidence also suggests that excess sedentary time is positively associated with metabolic risk factors, an effect that is independent of physical activity levels. ${ }^{15-17}$ Sedentary behaviours have been defined as any waking behaviour that is in a sitting, reclining or lying down posture, expending little energy (ie, 1.0 to 1.5 metabolic equivalents (METs) $).{ }^{18}$ Some studies have found that the cellular and molecular responses to sedentary time are different to the beneficial responses to physical activity. ${ }^{19}$ Therefore, it is essential to explore the effects of physical activity and sedentary time simultaneously, when exploring the associations between physical activity and sedentary time with metabolic risk factors. It is also crucial to elucidate whether this association is independent of sleep duration which might confound results. ${ }^{20}$

Furthermore, it has been suggested that using the continuous summary Score of Clustered Metabolic Risk $(\mathrm{zMS})^{11}$ for analyses may improve the statistical power to detect associations, compared with using a binary definition of dichotomising continuous outcome variables. ${ }^{21}$ Many studies have focused on the risk of metabolic syndrome in Western populations, ${ }^{9-131516}$ but the relationship between physical activity and sedentary time with the risk of metabolic syndrome is not well understood among Chinese populations. Although there have been a few studies in diabetic populations, their findings were inconsistent and limited by small sample sizes. ${ }^{10} 1117$ Therefore, it is interesting to explore the direction and strength of associations between physical activity and sedentary time with clustered metabolic risk factors among the Chinese population.

The objective of this study was to examine the association between total physical activity, physical activity in different domains (ie, occupation, commuting and leisure-time physical activity) and sedentary time with clustered and individual metabolic risk factors among Chinese patients with type 2 diabetes. We hypothesised that physical activity is inversely associated with clustered and individual metabolic risk factors, whereas sedentary time increases such risk.

\section{METHODS}

\section{Participants}

Participants were from the "Comprehensive Research on the Prevention and Control of the Diabetes" project. The method and study design have been previously described. ${ }^{22}$ Briefly, 29705 registered diabetic patients, receiving management from National Basic Public Health Service, were recruited from 44 selected townships across two cities in Jiangsu province, China. After excluding non-type 2 diabetic patients, and individuals with poor physical or mental status, a total of 20340 individuals consented to participate. In our analyses, we further excluded those with missing physical activity or sedentary time values $(\mathrm{n}=2038)$, those who reported spending $\geq 16$ hours on daily physical activity $(n=67),{ }^{23}$ and those with incomplete metabolic risk data $(n=198)$. Finally, there were 17750 participants included in the analyses.

\section{Assessment of physical activity and sedentary time}

Information on physical activity and sedentary time was collected using the Global Physical Activity Questionnaire (GPAQ). The validity and reliability of GPAQ has been assessed in Western $^{24}$ and Asian populations. ${ }^{25}$ Participants were asked to recall the intensity and duration of occupational, commuting and leisure-time physical activities over the previous weeks.

The intensity of activities was defined as moderate or vigorous by MET. ${ }^{26}$ Average MET scores were calculated based on specific activities within corresponding categories to estimate activity intensity (online supplementary table S1). The number of hours spent per day on each measured activity was multiplied by the corresponding average MET score and the measured activity frequency (days per week). Total physical activity was calculated by adding together the MET-hours for activities related to occupational, commuting and leisure-time domains. The average daily activity was calculated by dividing the weekly amount of physical activities by seven. Participants were also asked about their normal time spent on sedentary activities and sleep (hours/day).

\section{Clustered metabolic risk score}

We constructed a summary variable (zMS) for clustered metabolic risk ${ }^{11}$ that was broadly based on the definition proposed by Adult Treatment Panel III. ${ }^{2}$ This variable was calculated by adding the standardised values for waist circumference, fasting triacylglycerol, fasting plasma glucose, systolic blood pressure and the inverse of high-density lipoprotein cholesterol (HDL-cholesterol). Each of these variables was standardised by subtracting the sex-specific sample means from the individual mean and dividing by the SD. The zMS is a continuous variable 
with a mean of zero, by definition, with lower scores revealing a more favourable profile.

\section{Assessment of anthropometric, metabolic risk factors}

Face-to-face questionnaire interviews were also used to collect information on demographic characteristics, medical history, medications, smoking status, alcohol consumption and adult socioeconomic status. The detail of anthropometric measurements have been previously illustrated. ${ }^{27}$ Blood samples were collected in the morning following overnight fasting. Fasting plasma glucose was assessed using the Hexokinase method. Glycated haemoglobin (HbA1c) was measured in venous samples using high efficiency liquid chromatography. Serum cholesterol, HDL-cholesterol and triacylglycerol were measured enzymatically. All samples were analysed by KingMed Diagnostics (Jiangsu Cultural Industrial Park, Nanjing, China).

\section{Covariate definitions}

Covariates included age, sex, education (no formal education, primary, middle, high school or above), smoking status (yes, no), annual household income $(<30$ 000 RMB, 40 000-100 000 RMB, 110 000-150 000 RMB, $>160000 \mathrm{RMB}$ ), alcohol consumption (never, former, current), diabetes duration, taking medications to lower glucose and lipid or blood pressure (yes, no, unclear). Hypertension was defined as a systolic blood pressure $\geq 140 \mathrm{~mm} \mathrm{Hg}$ and/or a diastolic blood pressure $\geq 90 \mathrm{~mm}$ $\mathrm{Hg}$, or a previous hypertension diagnosis in a hospital. ${ }^{28}$ Dyslipidaemia was defined as having at least one of the following conditions: total cholesterol $\geq 5.2 \mathrm{mmol} / \mathrm{L}$, fasting blood triglyceride $\geq 1.7 \mathrm{mmol} / \mathrm{L}$, fasting blood low-density lipoprotein cholesterol $\geq 3.4 \mathrm{mmol} / \mathrm{L}$, HDL-cholesterol $<0.9 \mathrm{mmol} / \mathrm{L}$ for males or $<1.0 \mathrm{mmol} / \mathrm{L}$ for females, or previously being diagnosed with hyperlipidaemia in a hospital. ${ }^{29}$ Sleep duration was assessed by the question, 'How many cumulative hours do you have for sleep on an average day?'

\section{Statistical analyses}

Descriptive statistics were conducted separately for men and women using the mean $\pm \mathrm{SD}$, median (IQR) or frequencies. The $\chi^{2}$ test, Student's t-test, one-way analysis of variance and Mann-Whitney $\mathrm{U}$ test were used to examine the differences in characteristics between men and women. Fasting plasma glucose and triacylglycerol were logarithmically transformed (base e) due to their skewed distribution. Adjusted means and SE of clustered and individual metabolic risk variables were presented to explore the association between physical activity and sedentary time with metabolic risk. Test for trend was based on variables containing median value for each quartiles. Multiple comparisons among groups were performed using a Bonferroni method.

Associations between per 1 SD of total physical activity, different domains of physical activity (occupational, commuting and leisure-time) and sedentary time with total clustered metabolic risk score and individual metabolic risk factors were evaluated using multivariate linear regression. Preliminary checks were conducted to ensure no violation of assumptions of normality, homogeneity of variance and absence of multicollinearity. Four models were fitted: model 1 was unadjusted; model 2 was adjusted for age, sex, education, annual household income, smoking status, drinking and diabetes duration; model 3 was additionally adjusted for remaining physical activity and sedentary time, for each individual domain of physical activity was adjusted for the other physical activity domains and sedentary time as appropriate; model 4 was further adjusted for sleep duration. For models 2 to 4, all subcomponents except zMS and waist circumference were additionally adjusted for waist circumference. When the dependent variable was fasting plasma glucose, we additionally adjusted for the use of glucose-lowering medication. For triacylglycerol and HDL-cholesterol, we adjusted for the diagnosis of dyslipidaemia and use of lipid-lowering medication. When the outcome of interest was systolic blood pressure, we additionally adjusted for the diagnosis of hypertension and use of antihypertensive medication. For zMS we adjusted for the use of glucose-lowering, lipid-lowering or antihypertensive medications, and the diagnosis of dyslipidaemia and hypertension.

To investigate the joint associations of total physical activity and sedentary time with clustered metabolic risk, participants were classified into three and four groups of sex-specific tertiles of physical activity and quartiles of sedentary time, respectively. The adjusted zMS mean was calculated using general linear regression models after adjusting for age. Interactions between the main predictive variables, and sex and age (continuous) were examined by entering the centred interaction terms into multivariate linear regression models to determine whether the association between physical activity and sedentary time with metabolic risk was modified by sex and age. ${ }^{30}$ In further sensitivity analyses, we also calculated a metabolic syndrome score without the waist circumference in order to examine whether the associations between the main exposures (physical activity and sedentary time) and clustered metabolic risk were mediated by waist circumference.

All statistical analyses were performed using IBM SPSS Statistics standard V.23.0. Statistical significance was set at $\mathrm{p}<0.05$ for main effects, and $\mathrm{p}<0.10$ for interactions. Tests were two-sided.

\section{Patient and public involvement statement}

Research question development was informed by qualitative interviews, physical examinations and laboratory tests with a purposively selected sample of residents from the National Basic Public Health Services in Jiangsu province. Summary reports about the study results will be disseminated to participants, policy-makers and healthcare workers in the community (village doctors in rural areas or general practitioners in urban areas of China) through 
Table 1 Demographic, metabolic characteristics of participants

\begin{tabular}{|c|c|c|c|c|}
\hline characteristic & Men & Women & Total & P value \\
\hline $\mathrm{N}$ & 7041 & 10709 & 17750 & \\
\hline Age (years) & $62.6 \pm 9.9$ & $62.8 \pm 9.7$ & $62.8 \pm 9.8$ & 0.11 \\
\hline High school or above (\%) & 17.7 & 4.5 & 9.8 & $<0.001$ \\
\hline Annual income > $¥ 40000$ (\%) & 64.8 & 57.0 & 60.1 & $<0.001$ \\
\hline Body mass index $\left(\mathrm{kg} / \mathrm{m}^{2}\right)$ & $25.3 \pm 3.2$ & $25.4 \pm 3.6$ & $25.3 \pm 3.4$ & 0.09 \\
\hline Waist circumference (cm) & $87.9 \pm 9.4$ & $85.5 \pm 9.5$ & $86.4 \pm 9.5$ & $<0.001$ \\
\hline Systolic blood pressure $(\mathrm{mm} \mathrm{Hg})$ & $147.3 \pm 19.8$ & $148.9 \pm 21.1$ & $148.0 \pm 20.6$ & 0.002 \\
\hline Diastolic blood pressure $(\mathrm{mm} \mathrm{Hg})$ & $83.7 \pm 10.7$ & $79.9 \pm 10.4$ & $81.4 \pm 10.6$ & $<0.001$ \\
\hline Triacylglycerol $(\mathrm{mmol} / \mathrm{L})^{*}$ & $1.4(1.0,2.2)$ & $1.7(1.2,2.3)$ & $1.6(1.1,2.3)$ & $<0.001$ \\
\hline HDL-cholesterol (mmol/L) & $1.4 \pm 0.4$ & $1.5 \pm 0.4$ & $1.5 \pm 0.4$ & $<0.001$ \\
\hline Fasting plasma glucose $(\mathrm{mmol} / \mathrm{L})^{*}$ & $8.2(6.4,10.2)$ & $8.0(6.5,9.9)$ & $8.1(6.6,10.0)$ & $<0.001$ \\
\hline $\mathrm{HbA} 1 \mathrm{c}(\mathrm{mmol} / \mathrm{mol})^{\star}$ & $56.3(46.5,70.5)$ & $55.2(46.5,68.3)$ & $55.2(46.5,69.4)$ & $<0.001$ \\
\hline Diabetes duration (years) ${ }^{*}$ & $5(2,9)$ & $5(2,9)$ & $5(2,9)$ & 0.26 \\
\hline Smoking (\%) & 46.0 & 6.2 & 22.0 & $<0.001$ \\
\hline \multicolumn{5}{|l|}{ Alcohol consumption (\%) } \\
\hline Never & 49.1 & 96.6 & 77.7 & $<0.001$ \\
\hline Former & 10.7 & 1.1 & 4.9 & $<0.001$ \\
\hline Current & 40.1 & 2.3 & 17.4 & $<0.001$ \\
\hline On glucose-lowering medication (\%) & 78.7 & 78.4 & 78.5 & 0.60 \\
\hline On lipid-lowering medication (\%) & 5.8 & 5.9 & 6.0 & 0.005 \\
\hline On antihypertensive medication (\%) & 52.7 & 53.9 & 53.4 & 0.002 \\
\hline Hypertension (\%) & 77.5 & 76.6 & 77.0 & 0.16 \\
\hline Dyslipidaemia (\%) & 46.1 & 49.3 & 48.0 & $<0.001$ \\
\hline Total physical activity (MET hours/day) ${ }^{*}$ & $6.3(2.3,14.3)$ & $8.0(3.4,16.0)$ & $8.0(2.9,15.4)$ & $<0.001$ \\
\hline \multicolumn{5}{|l|}{ Physical activity domains (MET hours/day) $\dagger$} \\
\hline Occupational & $68.2(2.9(0.0,8.6))$ & $81.9(5.1(1.7,12.0))$ & $\begin{array}{l}76.5(4.0(0.6 \\
12.0))\end{array}$ & $<0.001 \ddagger$ \\
\hline Commuting & $59.4(1.1(0.0,1.3))$ & $62.0(1.0(0.0,2.9))$ & $60.1(1.0(0.0,2.9))$ & $<0.001 \ddagger$ \\
\hline Leisure times & $25.6(3.1(1.7,6.0))$ & $19.0(2.9(1.5,5.1))$ & $21.6(3.0(1.7,5.7))$ & $<0.001 \ddagger$ \\
\hline Sedentary time (hours/day) & $3.5 \pm 2.5$ & $3.4 \pm 2.6$ & $3.4 \pm 2.5$ & $<0.001$ \\
\hline Sleep duration (hours/day) & $7.4 \pm 1.6$ & $7.3 \pm 1.7$ & $7.4 \pm 1.7$ & $<0.001$ \\
\hline
\end{tabular}

$P$ values are from Student's t-test or Mann-Whitney $U$ test for continuous variables and from $\chi^{2}$ test for categorical variables.

Data are mean \pm SD unless stated otherwise.

*Median (IQR).

$\dagger(\%)$ Median (IQR).

$\ddagger P$ values are from $\chi^{2}$ test.

$\mathrm{HDL}$, high-density lipoprotein; HbA1c, glycated haemoglobin; MET, metabolic equivalent.

mass media, such as local newspapers, the internet, radios or workshops.

\section{RESULTS}

Demographic and basic characteristics of participants

Of the 17750 participants included in the analyses, $60.3 \%$ were women, and the average age of men and women was $62.6 \pm 9.9$ and $62.8 \pm 9.7$ years, respectively (table 1 ). Indices of body mass index, HDL-cholesterol and fasting plasma glucose did not differ significantly (all $\mathrm{p}>0.10$ ) between excluded ( $\mathrm{n}=2303)$ and included participants, but included participants had a slightly higher waist circumference and systolic blood pressure (all $\mathrm{p}<0.001$ ) (online supplementary table S2) than those who were excluded. Compared with women, men had a higher education level, household income and consumption of alcohol and cigarettes (all $\mathrm{p}<0.001$ ). Men also had higher waist circumference, $\mathrm{HbA1c}$, diastolic blood pressure and 
fasting plasma glucose levels than women (all $\mathrm{p}<0.001$ ). In contrast, women had higher HDL-cholesterol, systolic blood pressure and triacylglycerol levels than men (all $\mathrm{p}<0.001$ ).

Differences between sexes were also observed in physical activity, sedentary time and sleep duration. Women had higher total and occupational physical activity than men (all $\mathrm{p}<0.001$ ), whereas men had higher sedentary time, sleep duration, commuting and leisure-time physical activity than women $($ all $\mathrm{p}<0.001)$. The proportion of subjects participating in leisure-time physical activity was $21.6 \%$ (table 1).

\section{Adjusted means of clustered and individual metabolic variables by physical activity and sedentary time}

There was a tendency towards lower zMS, waist circumference, triacylglycerol and higher HDL-cholesterol at higher levels of total physical activity ( $p$ for trend $<0.001$ ) (table 2), while individuals with higher zMS, waist circumference, fasting plasma glucose, triacylglycerol and lower HDL-cholesterol were apt to have a higher sedentary time ( $p$ for trend $<0.001$ ) (table 2).

\section{Associations between physical activity and sedentary time with metabolic risk}

Total physical activity was inversely associated with zMS $(\mathrm{B}=-0.080 ; 95 \%$ CI: -0.114 to -0.046$)$, waist circumference $(\mathrm{B}=-0.449 ; 95 \% \mathrm{CI}:-0.591$ to -0.308$)$ and triacylglycerol ( $\mathrm{B}=-0.012 ; 95 \% \mathrm{CI}:-0.019$ to -0.006$)$, after adjusting for sedentary time and sleep duration. In contrast, the SD for physical activity was marginally positively associated with HDL-cholesterol ( $\mathrm{B}=0.009$; $95 \% \mathrm{CI}: 0.003$ to 0.015 ). No significant associations were found between total physical activity with fasting plasma glucose $(\mathrm{B}=0.004 ; 95 \% \mathrm{CI}$ : -0.001 to 0.008$)$ and systolic blood pressure $(B=-0.096$; 95\% CI: -0.346 to 0.155 ) (table 3).

In adjusted analyses, occupational physical activity was associated with zMS ( $\mathrm{B}=-0.066$; $95 \% \mathrm{CI}$ : -0.101 to $-0.031)$, waist circumference $(\mathrm{B}=-0.475 ; 95 \% \mathrm{CI}:-0.620$ to -0.334$)$, triacylglycerol $(\mathrm{B}=-0.007 ; 95 \% \mathrm{CI}:-0.014$ to $-0.001)$ and HDL-cholesterol ( $\mathrm{B}=0.006$; 95\% CI: 0.001 to 0.013$)$, but not with fasting plasma glucose $(\mathrm{B}=0.005$; 95\% CI: -0.001 to 0.009 ) and systolic blood pressure $(\mathrm{B}=0.081$; 95\% CI: -0.173 to 0.334$)$. Commuting physical activity was only inversely associated with triacylglycerol (B=-0.012; 95\% CI: -0.019 to -0.005$)$. Furthermore, leisure-time physical activity was found to be inversely associated with zMS ( $\mathrm{B}=-0.041 ; 95 \% \mathrm{CI}:-0.075$ to -0.007$)$ and systolic blood pressure ( $\mathrm{B}=-0.293$; $95 \%$ CI: -0.542 to $-0.045)$, but not with waist circumference, fasting plasma glucose or triacylglycerol (all p >0.05) (table 3 ).

Different from that of physical activity, sedentary time was strongly positively associated with zMS $(B=0.117$; 95\% CI: 0.083 to 0.151$)$ and waist circumference $(\mathrm{B}=0.474$; 95\% CI: 0.334 to 0.613 ), after adjustment for physical activity and sleep duration. Furthermore, sedentary time was found to be weakly associated with fasting plasma glucose ( $\mathrm{B}=0.008 ; 95 \%$ CI: 0.003 to 0.013$)$, triacylglycerol levels $(\mathrm{B}=0.015$; 95\% CI: 0.008 to 0.022$)$ and HDL-cholesterol ( $\mathrm{B}=-0.007$; $95 \%$ CI: -0.013 to -0.001 ). No significant association was observed between sedentary time and systolic blood pressure $(\mathrm{B}=0.215 ; 95 \% \mathrm{CI}:-0.033$ to 0.462) (table 3).

\section{Joint association of total physical activity and sedentary time with metabolic risk}

The joint association of total physical activity and sedentary time with zMS after adjustment for age is illustrated in figure 1. Participants with the lowest level of physical activity or the highest sedentary time were consistently found to have the highest zMS among both sexes. Interaction analyses indicated significant effects among different age groups, where greater associations with sedentary time, total and occupational physical activity for $\mathrm{zMS}(\mathrm{p}$ for interaction $<0.10$ ) and waist circumference ( $p$ for interaction $<0.05$ ) were observed in older age groups. In addition, there was no significant interaction with sex in the results of total physical activity, different domains of physical activity or sedentary time (online supplementary table S3). When excluding waist circumference from the zMS and additionally adjusting for waist circumference, the magnitude of association between sedentary time and clustered risk was weaker but remained statistically significant (online supplementary table S4).

\section{DISCUSSION}

In this cross-sectional study of a large sample-size of individuals with type 2 diabetes in China, we found that total physical activity, occupational physical activity and sedentary time were independently associated with clustered metabolic risk, waist circumference, triacylglycerol levels and HDL-cholesterol. Moreover, leisure-time physical activity was associated with systolic blood pressure and commuting physical activity was inversely associated with triacylglycerol. However, a higher level of sedentary time was positively associated with higher levels of fasting plasma glucose. Our findings suggest that increasing physical activity across all domains and decreasing amount of time spent sedentarily may have protective effects on reducing metabolic risk.

The finding of an association between total physical activity and clustered metabolic risk in this study is consistent with previous research findings with objective data. ${ }^{11} 12$ Importantly, we observed that occupational physical activity reduced the clustered metabolic risk in patients with type 2 diabetes. Occupational physical activity is a major source of total physical activity among Chinese and other Asian populations, ${ }^{14}$ for example, in this study, $75.8 \%$ of diabetic patients reported engaging in occupational physical activity. This suggests that occupational physical activity as a single domain may be important in the management of metabolic risk among Chinese or, perhaps more broadly, Asian populations. In a recent study, a trend was observed between lower levels of commuting physical activity and higher metabolic risk. ${ }^{31}$ 


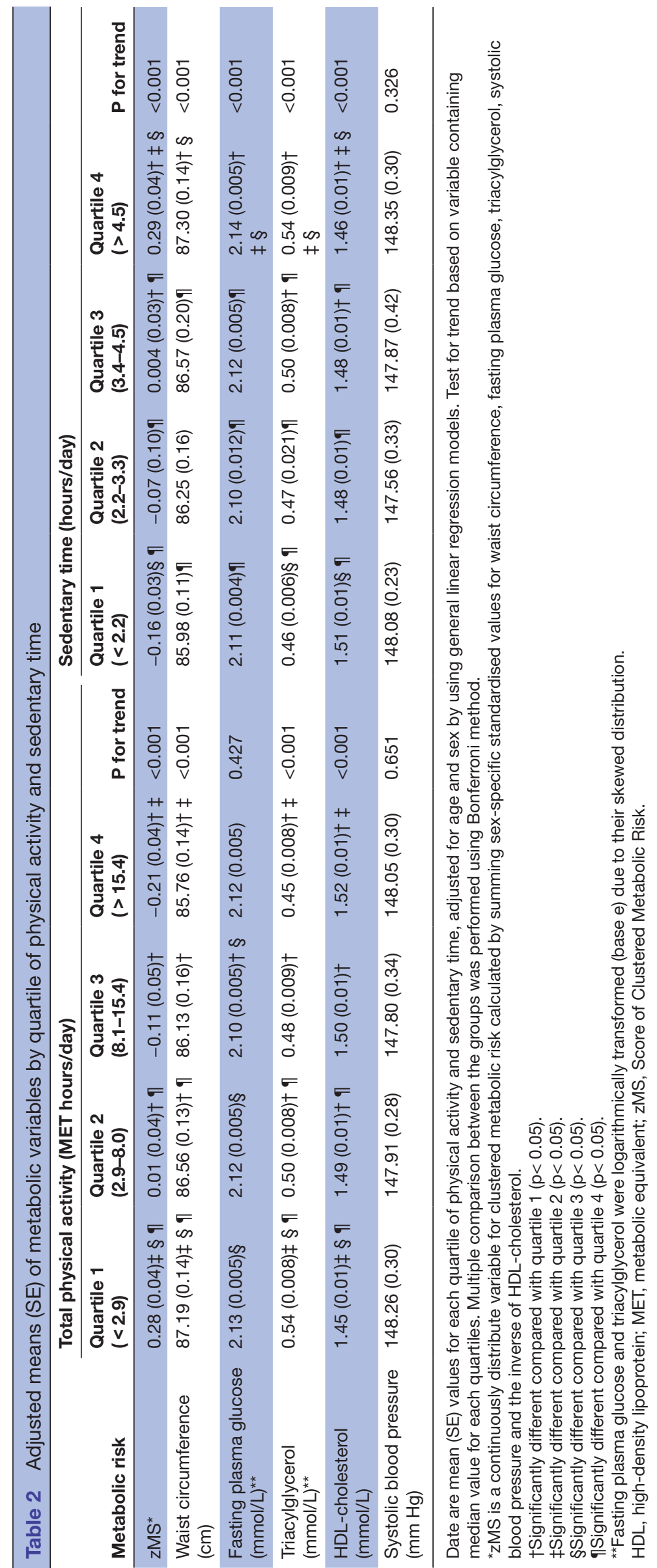

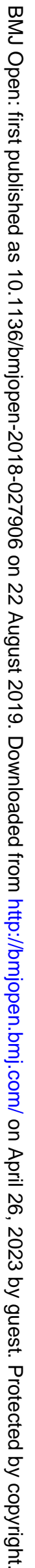




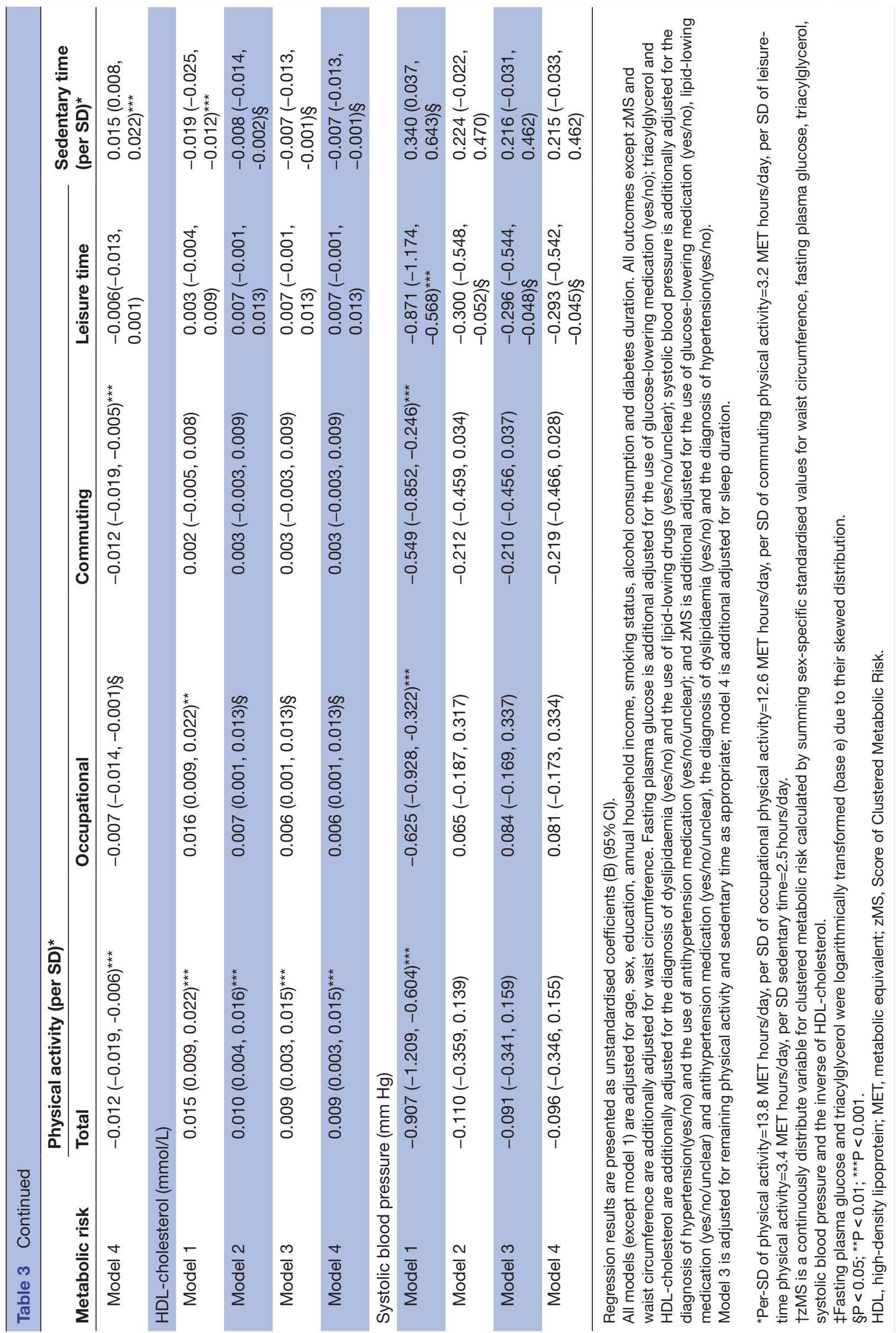




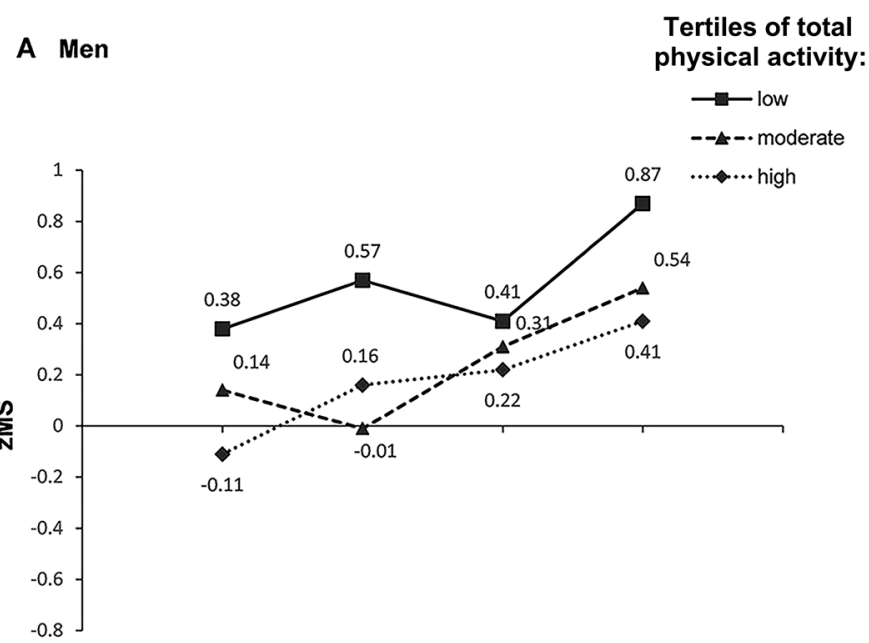

Quartiles of sedentary time

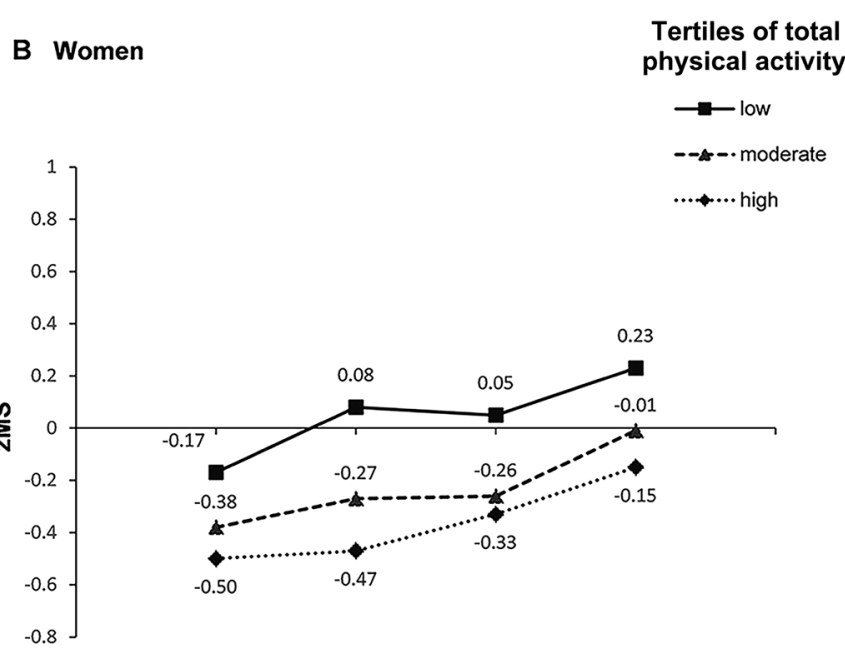

Quartiles of sedentary time

Figure 1 Means of clustered metabolic risk scores by sedentary time and physical activity among 17750 patients with diabetes. The adjusted mean was calculated using general linear regression models after adjustment for age. For physical activity, in men, the cut-off values for defining low, moderate and high tertile groups were 4.00 and $11.14 \mathrm{MET}$ hours/day, while they were 4.57 and $12.00 \mathrm{MET}$ hours/day for women. For sedentary time, the four groups by quartiles of sedentary time were $<2.2,2.3-3.3,3.4-4.9$ and $\geq 5.0$ hours/day for men and $<2.2,2.3-3.3,3.4-4.4$ and $\geq 4.5$ hours/day for women.

Our findings have also suggested that commuting physical activity is inversely associated with triacylglycerol, but there was no statistically significant association observed between commuting physical activity and clustered metabolic risk in this study, similar to the finding of the Japan Epidemiology Collaboration on Occupational Health study. ${ }^{32}$ This may partly be explained by the low level of commuting physical activity in our study, since $40 \%$ of patients reported that they did not engage in commuting physical activity.

Previous studies have also suggested that the associations between physical activity and clustered metabolic risk may be weakened by increasing sedentary time. ${ }^{1133}$ For example, a longitudinal study reported that the association between moderate-to-vigorous physical activity and clustered metabolic risk was attenuated when additionally adjusted for a 6-year change in sedentary time, indicating that focusing solely on physical activity may not be the most effective intervention strategy. ${ }^{33}$ In this study, we also found that sedentary time was associated with clustered and individual metabolic risk, which is independent of the effect of physical activity. This highlights the importance of not only increasing physical activity levels of patients with type 2 diabetes, but also reducing their sedentary time.

As reported previously, waist circumference is a body fat parameter most closely associated with metabolic syndrome. ${ }^{34}$ Our findings indicate that total and occupational physical activity are strongly associated with waist circumference. There is growing evidence to suggest that physical activity may support weight loss through regulation of adipokines (adiponectin, leptin, resistin, interleukin-6) which are known to contribute to cardiometabolic health. ${ }^{35}$ However, the biological mechanisms for associations between sedentary time and waist circumference with metabolic risk are not fully understood. One study has reported that this association can be attenuated once an adiposity component was included in the regression model.$^{36}$ Our findings suggested that more sedentary time was associated with a higher waist circumference, however, whether central obesity is a cause or a consequence of a sedentary behaviour has not yet been fully elucidated. ${ }^{37}$ Sedentary time may displace physical activity time, leading to a decrease in energy expenditure and unfavourable weight changes. ${ }^{38}$ Additional pathways linking sedentary time, waist circumference and metabolic risk include the established associations between major sedentary behaviour (television viewing) and an unhealthy diet. ${ }^{39}$ In addition, when excluding waist circumference from the clustered metabolic risk score and adjusting for it as a confounding factor, the magnitude of association between physical activity and sedentary time with clustered metabolic risk was attenuated. An alternative explanation is that waist circumference might be an important part of the causal pathway between sedentary time and clustered metabolic risk, but further research is needed. We also found that the relationship between sedentary time with zMS and waist circumference was modified by age. This may be partly due sedentary time increasing with age. ${ }^{40}$ Older patients may have a higher chance of clustered metabolic risk than younger people based on this age-related increase of sedentary time.

The association between total and individual domains of physical activity with fasting plasma glucose did not reach statistical significance in this study, which is consistent with result from the ProActive Study. ${ }^{12}$ In contrast, a 
significant association was found between sedentary time and fasting plasma glucose in our study. The potential mechanism between sedentary time and fasting plasma glucose involves markers of inflammation, insulin resistance and adiposity. ${ }^{41}$ Obesity has a proven association with the increased risk of developing insulin resistance, and may result in disordered regulation of glucose levels by reducing insulin release. ${ }^{42}$ Furthermore, another study has suggested that sleep loss can lead to disordered glucose metabolism. ${ }^{43}$ In our study, we obtained consistent results even after adjusting for sleep duration, suggesting that the relationship between sedentary time and metabolic risk is independent of sleep duration.

In the present analysis, non-significant results between total physical activity, occupational physical activity and sedentary time with systolic blood pressure are consistent with the results from most previous studies, ${ }^{11} 12173132$ Moreover, our study identified a significant inverse association between leisure-time physical activity and systolic blood pressure. Clays et al also observed that objectively measured moderate and vigorous leisure-time physical activity, but not occupational physical activity, were inversely associated with systolic blood pressure. ${ }^{44} \mathrm{~A}$ further study showed that bouts of high-intensity physical activity elicited greater systolic blood pressure reductions than lower-intensity bouts. ${ }^{45}$ However, practice recommendations advocating high-intensity physical activity as an antihypertensive therapy is challenging because adverse cardiovascular effects are more likely to occur with vigorous than moderate to low levels of physical exertion, ${ }^{46}$ especially in patients with type 2 diabetes who have a significantly greater systolic blood pressure response to a given physical activity intensity than that of the healthy population. ${ }^{47}$ The American Diabetes Association (ADA) recommended that patients with type 2 diabetes should be assessed for conditions that might be associated with risk of cardiovascular disease before undertaking vigorous physical activity. ${ }^{48}$ Our findings also suggest that patients with type 2 diabetes should comply with ADA recommendations to increase overall physical activity through work, active transport and participation in physical activity, as well as reducing sedentary behaviour to lower metabolic risk.

This study has several strengths. A relatively large sample size $(\mathrm{n}=17750)$ was used to explore the association between physical activity, sedentary time and metabolic risk in a Chinese population. To the best of our knowledge, this is the first study in China that has evaluated physical activity in different domains and sedentary time with metabolic risk factors in patients with type 2 diabetes. Several limitations should also be mentioned. First, as a cross-sectional study, the direction and causality of the associations obtained from this study were restricted to inference. Second, the waist circumference and systolic blood pressure of included participants were slightly higher than those of excluded cases which may reduce the accuracy of the estimation of our results. Third, all participants were recruited from just two areas in Jiangsu province, China, so whether these findings can be generalised to the population at large still needs to be discussed. Finally, a self-reporting questionnaire was used to acquire information on physical activity, so reporting errors may have occurred due to a recall bias. Self-reported measures of physical activity are easier to use but they may have limited validity when compared with objective measures. ${ }^{49}$ Moreover, the patterns of physical activity and sedentary behaviours may vary between weekdays and weekends, but such variation is hard to obtain through the self-reporting measures used in this study. Further research is needed to explore a more detailed relationship between different patterns of physical activity and sedentary behaviour (specifically comparing weekdays and weekends) with metabolic risk in patients with type 2 diabetes.

\section{CONCLUSIONS}

The findings of this study suggest that total physical activity, physical activity in different domains and sedentary time are associated with clustered and individual metabolic risk factors in community-managed patients with type 2 diabetes. Encouraging patients with type 2 diabetes to increase physical activity in different domains and decrease sedentary time may have protective effects against metabolic risk. Therefore, the findings from this study extend previous cross-sectional evidence of associations between physical activity, sedentary time and metabolic risk in the Chinese population.

\section{Author affiliations}

${ }^{1}$ Department of Epidemiology and Health Statistics, School of Public Health, Southeast University, Nanjing, China

${ }^{2}$ Department of Non-communicable Chronic Disease Control, Jiangsu Provincial Centre for Disease Control and Prevention, Nanjing, China

${ }^{3}$ Department of Epidemiology, School of Public Health, Nanjing Medical University, Nanjing, China

${ }^{4}$ Department of Chronic Disease Prevention and Control, Huai'an Center for Disease Control and Prevention, Huai'an, China

${ }^{5}$ Institute of Suzhou Biobank, Suzhou Center for Disease Control and Prevention, Suzhou, China

${ }^{6}$ Changshu Center for Disease Control and Prevention, Changshu, China

Acknowledgements The authors thank all participants for their voluntary participation as well as the staff of local Health Bureaus and Centers for Disease Control and Prevention in cities of Changshu and Huai'an for their hard work and assistance during data collection. We would like to thank Editage (www.editage.cn) for English language editing.

Contributors MW and CS designed this study. JY, JS, YQ, CS, YL, LSL, EP, YG, DM, $\mathrm{NZ}, \mathrm{JZ}$ and MW were responsible for data collection. YC analysed the data and drafted the manuscript. MW, YQ, CS and EP revised the manuscript.

Funding This study was supported by grants from Jiangsu Province Leading Talents and Innovation Team Program (grant numbers K201105), Jiangsu Provincial Fourth "333 Project" (grant numbers BRA201307), National Natural Science Foundation of China (grant numbers 81602919).

Competing interests None declared.

Patient consent for publication Obtained.

Ethics approval The study protocol was approved by the Ethics Board of Jiangsu Provincial Centers for Disease Control and Prevention (reference number: 2013026).

Provenance and peer review Not commissioned; externally peer reviewed. 
Data availability statement Data are available upon reasonable request.

Open access This is an open access article distributed in accordance with the Creative Commons Attribution Non Commercial (CC BY-NC 4.0) license, which permits others to distribute, remix, adapt, build upon this work non-commercially, and license their derivative works on different terms, provided the original work is properly cited, appropriate credit is given, any changes made indicated, and the use is non-commercial. See: http://creativecommons.org/licenses/by-nc/4.0/.

\section{REFERENCES}

1. Eckel RH, Grundy SM, Zimmet PZ. The metabolic syndrome. The Lancet 2005;365:1415-28.

2. Grundy SM, Cleeman JI, Daniels SR, et al. Diagnosis and management of the metabolic syndrome: an American heart Association/National heart, lung, and blood Institute scientific statement. Circulation 2005;112:2735-52.

3. Li R, Li W, Lun Z, et al. Prevalence of metabolic syndrome in mainland China: a meta-analysis of published studies. BMC Public Health 2016;16:296.

4. Lu B, Yang $Y$, Song $X$, et al. An evaluation of the International diabetes Federation definition of metabolic syndrome in Chinese patients older than 30 years and diagnosed with type 2 diabetes mellitus. Metabolism 2006;55:1088-96.

5. Laaksonen DE, Lakka H-M, Niskanen LK, et al. Metabolic syndrome and development of diabetes mellitus: application and validation of recently suggested definitions of the metabolic syndrome in a prospective cohort study. Am J Epidemiol 2002;156:1070-7.

6. Lakka H-M, Laaksonen DE, Lakka TA, et al. The metabolic syndrome and total and cardiovascular disease mortality in middle-aged men. JAMA 2002;288:2709-16.

7. Girman CJ, Rhodes T, Mercuri M, et al. The metabolic syndrome and risk of major coronary events in the Scandinavian simvastatin survival study (4S) and the air Force/Texas coronary atherosclerosis prevention study (AFCAPS/TexCAPS). Am J Cardiol 2004;93:136-41.

8. Lakka TA, Laaksonen DE. Physical activity in prevention and treatment of the metabolic syndrome. Appl Physiol Nutr Metab 2007;32:76-88.

9. Boulé NG, Haddad E, Kenny GP, et al. Effects of exercise on glycemic control and body mass in type 2 diabetes mellitus: a metaanalysis of controlled clinical trials. JAMA 2001;286:1218-27.

10. Gordon LA, Morrison EY, McGrowder DA, et al. Effect of exercise therapy on lipid profile and oxidative stress indicators in patients with type 2 diabetes. BMC Complement Altern Med 2008;8:21.

11. Cooper AJM, Brage S, Ekelund U, et al. Association between objectively assessed sedentary time and physical activity with metabolic risk factors among people with recently diagnosed type 2 diabetes. Diabetologia 2014;57:73-82.

12. Ekelund U, Griffin SJ, Wareham NJ, et al. Physical activity and metabolic risk in individuals with a family history of type 2 diabetes. Diabetes Care 2007;30:337-42.

13. Kwaśniewska M, Kaczmarczyk-Chałas K, Pikala M, et al. Commuting physical activity and prevalence of metabolic disorders in Poland Prev Med 2010;51:482-7.

14. Ng SW, Popkin BM. Time use and physical activity: a shift away from movement across the globe. Obes Rev 2012;13:659-80.

15. Healy GN, Wijndaele K, Dunstan DW, et al. Objectively measured sedentary time, physical activity, and metabolic risk: the Australian diabetes, obesity and lifestyle study (AusDiab). Diabetes Care 2008;31:369-71.

16. Knaeps S, De Baere S, Bourgois J, et al. Substituting sedentary time with light and moderate to vigorous physical activity is associated with better cardiometabolic health. J Phys Act Health 2018;15:197-203.

17. Cooper AR, Sebire S, Montgomery AA, et al. Sedentary time, breaks in sedentary time and metabolic variables in people with newly diagnosed type 2 diabetes. Diabetologia 2012;55:589-99.

18. Sedentary Behaviour Research Network. Letter to the editor: standardized use of the terms "sedentary" and "sedentary behaviours". Appl Physiol Nutr Metab 2012;37:540-2.

19. Katzmarzyk PT, Activity P, Behavior S. And health: paradigm paralysis or paradigm shift? Diabetes 2010;59:2717-25.

20. Troxel WM, Buysse DJ, Matthews KA, et al. Sleep symptoms predict the development of the metabolic syndrome. Sleep 2010;33:1633-40.

21. Douglas GA, Patrick R. The cost of dichotomising continuous variables. BMJ 2006;323.
22. Miao DD, Pan EC, Zhang Q, et al. Development and validation of a model for predicting diabetic nephropathy in Chinese people. Biomed Environ Sci 2017;30:106-12.

23. FanM, lyuJ, HeP. Chinese guidelines for data processing and analysis concerning the International physical activity questionnaire. Chin $J$ Epidemiol 2014;35:961-4.

24. Bull FC, Maslin TS, Armstrong T. Global physical activity questionnaire (GPAQ): nine country reliability and validity study. $J$ Phys Act Health 2009;6:790-804.

25. AHY C, SHX N, Koh D, et al. Domain-Specific adult sedentary behaviour questionnaire (ASBQ) and the GPAQ single-item question: a reliability and validity study in an Asian population. Int $J$ Environ Res Public Health 2018;15.

26. Ainsworth BE, Haskell WL, Whitt MC, et al. Compendium of physical activities: an update of activity codes and Met intensities. Med Sci Sports Exerc 2000;32(9 Suppl):S498-S516.

27. Wu M, Wen J, Qin Y, et al. Familial history of diabetes is associated with poor glycaemic control in type 2 diabetics: a cross-sectional study. Sci Rep 2017;7:1432.

28. Chobanian AV, Bakris GL, Black HR, et al. Seventh report of the joint National Committee on prevention, detection, evaluation, and treatment of high blood pressure. Hypertension 2003;42:1206-52.

29. Huang Y, Gao L, Xie X, et al. Epidemiology of dyslipidemia in Chinese adults: meta-analysis of prevalence, awareness, treatment, and control. Popul Health Metr 2014;12:2-9.

30. Aiken LS, West SG. Multiple regression: testing and interpreting interactions. Newbury Park California: SAGE Publications, 1991: 116-37.

31. AHY C, Moy FM. Associations of occupational, transportation, household and leisure-time physical activity patterns with metabolic risk factors among middle-aged adults in a middle-income country. Pre Med 2013;57:S14-S17.

32. Kuwahara K, Honda T, Nakagawa T, et al. Leisure-Time exercise, physical activity during work and commuting, and risk of metabolic syndrome. Endocrine 2016;53:710-21.

33. Wijndaele K, Orrow G, Ekelund U, et al. Increasing objectively measured sedentary time increases clustered cardiometabolic risk: a 6 year analysis of the ProActive study. Diabetologia 2014;57:305-12.

34. Carr DB, Utzschneider KM, Hull RL, et al. Intra-Abdominal fat is a major determinant of the National cholesterol education program adult treatment panel III criteria for the metabolic syndrome. Diabetes 2004;53:2087-94.

35. Vella CA, Allison MA, Cushman M, et al. Physical activity and Adiposity-related inflammation: the MESA. Med Sci Sports Exerc 2017;49:915-21.

36. Stamatakis E, Pulsford RM, Brunner EJ, et al. Sitting behaviour is not associated with incident diabetes over 13 years: the Whitehall II cohort study. Br J Sports Med 2017;51:818-23.

37. Ekelund $\mathrm{U}$, Brage $\mathrm{S}$, Besson $\mathrm{H}$, et al. Time spent being sedentary and weight gain in healthy adults: reverse or bidirectional causality? Am J Clin Nutr 2008;88:612-7.

38. Mekary RA, Willett WC, Hu FB, et al. Isotemporal substitution paradigm for physical activity epidemiology and weight change. Am J Epidemiol 2009;170:519-27.

39. Pearson N, Biddle SJH. Sedentary behavior and dietary intake in children, adolescents, and adults. A systematic review. Am J Prev Med 2011;41:178-88.

40. Matthews CE, Chen KY, Freedson PS, et al. Amount of time spent in sedentary behaviors in the United States, 2003-2004. Am J Epidemiol 2008;167:875-81

41. Yates T, Khunti K, Wilmot EG, et al. Self-Reported sitting time and markers of inflammation, insulin resistance, and adiposity. Am J Prev Med 2012;42:1-7.

42. Kahn SE, Hull RL, Utzschneider KM. Mechanisms linking obesity to insulin resistance and type 2 diabetes. Nature 2006;444:840-6.

43. Rafalson L, Donahue RP, Stranges S, et al. Short sleep duration is associated with the development of impaired fasting glucose: the Western New York health study. Ann Epidemiol 2010;20:883-9.

44. Clays E, De Bacquer D, Van Herck K, et al. Occupational and leisure time physical activity in contrasting relation to ambulatory blood pressure. BMC Public Health 2012;12:1002.

45. Eicher JD, Maresh CM, Tsongalis GJ, et al. The additive blood pressure lowering effects of physical activity intensity on postphysical activity hypotension. Am Heart J 2010;160:513-20.

46. Thompson PD, Franklin BA, Balady GJ, et al. Exercise and acute cardiovascular events placing the risks into perspective: a scientific statement from the American heart association Council on nutrition physical activity, and metabolism and the Council on clinical cardiology. Circulation 2007;115:2358-68. 
47. Brett SE, Ritter JM, Chowienczyk PJ. Diastolic blood pressure changes during exercise positively correlate with serum cholesterol and insulin resistance. Circulation 2000;101:611-5.

48. American Diabetes Association. 4. Lifestyle Management: Standards of Medical Care in Diabetes-2018. Diabetes Care 2018;41(Suppl 1):S38-S50.
49. Skender S, Ose J, Chang-Claude J, et al. Accelerometry and physical activity questionnaires - a systematic review. BMC Public Health 2016;16:515. 